\title{
A cohort study of unstable overdose patients treated with intravenous lipid emulsion therapy
}

\author{
Shazma Mithani, $\mathrm{MD}^{* *}$; Kathryn Dong, $\mathrm{MD}^{* *}$; Ashlea Wilmott, $\mathrm{MD}^{\S}$; Heather Podmoroff, $\mathrm{BN}, \mathrm{BScN}^{* *}$; \\ Nadim Lalani, MD ${ }^{\ddagger \neq}$; Rhonda J. Rosychuk, $\mathrm{PhD}^{\dagger}$; Ryan Chuang, MD ${ }^{\S * *}$; Mark C. Yarema, MD ${ }^{\S^{\dagger \dagger \dagger}}$
}

\section{ABSTRACT}

Objectives: Intravenous lipid emulsion (ILE) has been used increasingly over the last decade for a range of drug overdoses. Although the use of ILE in local anesthetic toxicity (LAST) is well established, the hemodynamic effectiveness of ILE in non-LAST poisonings is still unclear. Thus, the primary objective of this study was to examine a cohort of poisoned patients in whom ILE was administered.

Methods: Consecutive patients were identified by calls to a regional poison center from May 1, 2012 to May 30, 2014. Patients were enrolled if they ingested a drug, developed hemodynamic instability, failed conventional treatment, and received ILE therapy. Data were collected by medical record review. The primary outcome was the change in mean arterial pressure (MAP) in the first hour after ILE administration. Secondary outcomes included survival, length of stay, and the effect of drug class on patient outcome.

Results: Thirty-six patients were enrolled. Agents ingested included calcium channel blockers and beta blockers (10/36, $27.8 \%)$, tricyclic antidepressants $(5 / 36,13.9 \%)$, bupropion $(3 / 36$, $8.3 \%)$, and antiepileptic agents $(1 / 36,2.8 \%)$. Seventeen patients $(47.2 \%)$ ingested multiple agents. Twenty-five patients survived (69.0\%). Overall, MAP increased by $13.79 \mathrm{~mm} \mathrm{Hg}$ (95\% Cl $1.43-$ 26.15); this did not meet our a priori definition of clinical significance.

Conclusions: Our study did not find a clinically important improvement in MAP after ILE administration. Until future research is done to more definitively study its efficacy, ILE should remain a potential treatment option for hemodynamically unstable overdose patients only after conventional therapy has failed.

\section{RÉSUMÉ}

Objectif: Le recours à l'émulsion lipidique intraveineuse (ELI) a connu une augmentation au cours de la dernière décennie dans le traitement de divers types de surdosage. Si I'utilisation de l'ELI dans le traitement de la toxicité des anesthésiques locaux est bien établie, l'efficacité hémodynamique de I'ELI dans les cas d'intoxication causée par d'autres substances que les anesthésiques locaux, elle, est incertaine. L'étude décrite ici avait donc pour objectif principal d'examiner une cohorte de patients intoxiqués, traités par une ELI.

Méthode: Le repérage des patients consécutifs s'est fait à l'aide des appels reçus dans un centre antipoisons régional, du 1er mai 2012 au 30 mai 2014. Ont été retenus ceux qui avaient pris des médicaments, présentaient de l'instabilité hémodynamique, n'avaient pas réagi au traitement habituel et avaient été traités par une ELI. La collecte de données a été réalisée à I'aide des dossiers médicaux. Le principal critère d'évaluation consistait en la mesure des variations de la pression artérielle moyenne (PAM) au cours de la première heure suivant l'administration de I'ELI; les critères secondaires d'évaluation comprenaient la survie, la durée du séjour et l'effet de la classe de médicaments sur l'évolution de l'état de santé.

Résultats: Ont participle à l'étude 36 patients. Différents types de médicaments avaient été avalés, notamment des inhibiteurs calciques et des bêta-bloquants (10/36; 27,8 \%), des antidépresseurs tricycliques (5/36; 13,9\%), du bupropion (3/36; 8,3\%) et des antiépileptiques (1/36; $2,8 \%$ ). Dix-sept patients $(47,2 \%)$ avaient pris un mélange de médicaments. Vingt-cinq patients ont survécu $(69,0 \%)$ au surdosage. La PAM a augmenté, dans I'ensemble, de 13,79 mm Hg (IC à $95 \%$ : 1,43 - 26,15), mais l'écart ne respectait la définition de portée clinique, établie avant l'étude.

Conclusions: Les résultats de l'étude ne permettent pas de conclure à une amélioration importante de la PAM sur le plan clinique après l'administration de I'ELI. Aussi le traitement devrait-il rester une solution de rechange possible dans les seuls cas de surdosage accompagnés d'instabilité hémodynamique, après l'échec du traitement courant, jusqu'à ce que des recherches ultérieures examinent davantage en profondeur la question de l'efficacité de I'ELI.

Keywords: lipid emulsion, overdose, resuscitation, antidote

From the *Department of Emergency Medicine and †Department of Pediatrics, University of Alberta, Edmonton, AB; $¥$ Royal Alexandra Hospital, Alberta Health Services, Edmonton, AB; §Department of Emergency Medicine and ๆDepartment of Medicine, University of Calgary, Calgary, $A B$; **Poison and Drug Information Service and t†Section of Clinical Pharmacology and Toxicology, Alberta Health Services, Calgary, AB; and ¥¥Department of Emergency Medicine, University of Saskatchewan, Saskatoon, SK.

Correspondence to: Dr. Shazma Mithani, Department of Emergency Medicine, Royal Alexandra Hospital, 10240 Kingsway Avenue, Community Services Building, Room 541, Edmonton, AB, Canada, T5H 3V9; email: smithani@ualberta.ca 


\section{INTRODUCTION}

\section{Background}

The first use of intravenous lipid emulsion (ILE) therapy in the overdose setting was in 1998 in a rat model of bupivacaine toxicity. ${ }^{1}$ Since then, there have been multiple animal studies and human reports on the use of ILE in local anesthetic toxicity (LAST) ${ }^{2-8}$ ILE has now been incorporated into anesthesia practice guidelines for management of LAST.

A widely accepted mechanism of action for ILE is the "lipid sink" model. ${ }^{10,11}$ ILE is thought to expand the plasma lipid phase and act as a sink for lipophilic toxins, drawing them away from their target tissue receptors. In vitro studies suggest that the efficacy of ILE is greater with increasing drug lipophilicity (as measured by its volume of distribution and its partition constant). ${ }^{12}$ Based on these results, multiple cardiovascular toxins, including amiodarone, tricyclic antidepressants (TCAs), bupropion, verapamil, amlodipine, propranolol, and quetiapine could be amendable to ILE therapy. Other possible mechanisms include the alteration of fatty acid metabolism in cardiac myocytes and promotion of the opening of voltage-gated calcium channels in cardiac myocytes. ${ }^{13,14}$ The end result of the latter two mechanisms is an increase in cardiac inotropy (increased contractility) and chronotropy (increased heart rate).

\section{Importance}

In the last decade, the use of ILE for non-local anesthetic agent toxicity has been growing in both the emergency department (ED) and intensive care unit (ICU) settings. Multiple animal studies have demonstrated the efficacy of ILE for toxicity from beta blockers (BBs), calcium channel blockers (CCBs), TCAs, and antipsychotics. ${ }^{15-21}$

To date, the human data on the effectiveness of ILE for cardiovascular toxins has been limited to small case series and reports, and review articles. ${ }^{22-32} \mathrm{~A}$ recently published systematic review on the use of ILE in nonlocal anesthetic overdoses found current evidence to be heterogeneous and low quality. ${ }^{33}$ Moreover, several publications have demonstrated adverse effects with its use, including interference with laboratory investigations such as the complete blood count (CBC) and electrolytes. $^{34-43}$

Although ILE use is well established in LAST, the effectiveness of ILE for non-LAST poisonings is still unclear. The current literature on ILE is subject to publication bias and is likely to include a disproportionate number of positive outcome case reports.

\section{Objectives}

We sought to determine the effect of ILE on a consecutive cohort of poisoned patients in whom ILE was administered. Key outcomes of interest were the effect of ILE on mean arterial pressure (MAP), survival, time in hospital, and adverse events.

\section{METHODS}

\section{Study design and setting}

This was an observational study that enrolled consecutive patients from 16 Canadian tertiary care and community hospitals in four major cities within two Canadian provinces. Data were collected between May 1, 2012 and May 30, 2014. The study took place in partnership with the regional poison center, which covers a catchment area of 5.2 million people and fields over 34,000 calls annually (2013 data).

\section{Study population}

We wished to study patients who met accepted criteria for administration of ILE after a drug poisoning, namely: a) exposure to a lipophilic cardiotoxic drug, b) hemodynamic instability or cardiac arrest, and c) failure of routine supportive care to improve hemodynamics. ${ }^{44}$ To achieve this, we enrolled patients of all ages with exposure to any drug or combination of drugs with any of the following characteristics: cardiac arrest, systolic blood pressure (SBP) $<90 \mathrm{~mm} \mathrm{Hg}$, diastolic blood pressure $(\mathrm{DBP})<60 \mathrm{~mm} \mathrm{Hg}$, heart rate $(\mathrm{HR})<50 \mathrm{bpm}$, altered mental status, or seizure activity. Pediatric values for HR, DBP, and SBP were age-based, and are listed in Appendix 1. In addition, patients had to have received conventional treatment for their exposure (e.g., intravenous fluids, vasopressors, inotropes, or specific antidotes), without clinical improvement. The Certified Specialists in Poison Information (CSPI) at the poison center enrolled the patient in the study once the treating health care provider contacted the poison center for advice. Although there were no specific exclusion criteria, cases not reported to the poison center could not be identified. 


\section{Data collection}

Initial clinical and laboratory data were collected over the telephone using a standardized data collection form. These data included patient demographics, drug exposure, initial vital signs, treatment prior to ILE, and initial ILE dose. Additional information, including ILE infusion dose, vital signs after ILE administration, and patient outcome data were collected by medical record review by three of the authors (SM, AW, and NL). Data were then transferred onto a Microsoft Excel spreadsheet (Microsoft Corp., Redmond, WA) and reviewed by two of the authors (SM and $\mathrm{KD}$ ) for any errors or omissions.

\section{Interventions}

The recommended dosing protocol for ILE by our poison center during the study was a $1.5 \mathrm{~mL} / \mathrm{kg}$ bolus (which could be repeated up to three times), followed by an infusion of $0.25-0.50 \mathrm{~mL} / \mathrm{kg} /$ minute until a total ILE dose of $8 \mathrm{~mL} / \mathrm{kg}$ was reached. ${ }^{45}$ Patients who received variations of this protocol were included in the study. During the data collection period, some centers changed their ILE formulation from Intralipid ${ }^{\circledR}(20 \%$ soybean oil, $1.2 \%$ egg yolk phospholipids, $2.25 \%$ glycerin) to Clinoleic ${ }^{\circledR}$ (16\% olive oil, 4\% soybean oil, $1.2 \%$ egg yolk phospholipids, $2.25 \%$ glycerin). Patients receiving either formulation were included in the study.

\section{Outcome measures}

The primary outcome was the change in MAP over time following the administration of ILE. Our group felt that a clinically meaningful increase in MAP would be an improvement of at least $10 \mathrm{~mm} \mathrm{Hg}$ in the first hour after ILE administration, based on a study that demonstrated an increase in MAP by $10 \mathrm{~mm} \mathrm{Hg}$ in healthy volunteers given ILE. ${ }^{46}$ Secondary outcomes were survival, time from initiation to discontinuation of all vasopressors and inotropes after ILE administration, hospital length of stay, ICU length of stay, effect of drug class on patient outcome, and return to baseline neurologic status (defined as a return to pre-hospital neurologic function and assessed based on chart review of neurology referral/consultation and/or involvement of rehabilitation services). Data were also evaluated for evidence of lipemia interfering with the measurement of laboratory tests.

\section{Analysis}

MAP values after ILE administration were plotted to show the variability in individual patient measurements. The primary analysis used mixed effects models to quantify MAP after ILE administration with the patient as the random effect; time since baseline, age group, and ingested drug class were treated as fixed effects. This analysis allowed for multiple and varying measurement times per patient. All patients in whom a mean arterial pressure could not be obtained (e.g., pulseless, or systolic but no diastolic blood pressure recorded) were assigned a value of zero $\mathrm{mm} \mathrm{Hg}$ at that time point for all statistical calculations. Estimates over time and associated 95\% confidence intervals (CIs) were calculated and the estimated change in MAP at one hour was provided. In addition, the change in MAP at one hour after ILE administration was calculated and a one-sided $t$-test assessed if the change was at least $10 \mathrm{~mm} \mathrm{Hg}$. Patients with at least one measurement between baseline and one hour were included in this $t$-test; the last value was carried forward if a measurement was not available at one hour. Medians and quartiles were calculated for the time-to-event variables: time from initiation to discontinuation of all vasopressors and inotropes after ILE administration, hospital length of stay, and ICU length of stay. Analyses were repeated for the subgroups of survivors and non-survivors. Analyses were conducted using The R Project for Statistical Computing (https://www. r-project.org) using the NLME package. ${ }^{47} \mathrm{~A} p$-value of less than 0.05 was considered statistically significant.

\section{Ethical issues}

The study was approved by the Health Research Ethics Boards at our respective institutions (University of Alberta Health Research Ethics Board, University of Calgary Conjoint Health Research Ethics Board, and University of Saskatchewan Biomedical Research Ethics Board). Given the expected unstable clinical status of the patients on presentation, the study was granted a waiver of consent by all involved ethics boards.

\section{RESULTS}

A total of 36 patients were enrolled during the study period. The ingested agents included CCBs and BBs (10/36, 27.8\%), TCAs (5/36, 13.9\%), bupropion (3/36, $8.3 \%)$, and antiepileptic agents (1/36, 2.8\%). Seventeen 
patients (47.2\%) ingested agents from multiple classes (including selective serotonin reuptake inhibitors, antipsychotics, opioids, sedative hypnotics, sympathomimetics, clonidine, and valproic acid), whereas the remainder ingested agents from a single class. Table 1 describes the characteristics of the study group.

All patients were treated with intravenous fluid resuscitation, and 35 (97.2\%) patients were treated with additional agents to support hemodynamics prior to ILE administration. These agents included vasopressor infusions, bolus epinephrine as a part of advanced cardiac life support (ACLS) for cardiac arrest, atropine, sodium bicarbonate, calcium, glucagon, and high dose insulin with euglycemia (HIE) (Table 1). Dosing of glucagon (2 - $10 \mathrm{mg}$ bolus), calcium $(0.5-1 \mathrm{mEq} / \mathrm{kg})$, and $\mathrm{HIE}$ ( 1 unit $/ \mathrm{kg}$ insulin bolus) followed poison center recommendations in all but one glucagon patient.

There was variability in the ILE dosing regimens. Twenty-four $(66.7 \%)$ patients received an initial bolus between $1.0-2.0 \mathrm{~mL} / \mathrm{kg}$ of ILE. Nine patients $(25.0 \%)$ received a non-weight based bolus of between $50-400 \mathrm{~mL}$. Two patients $(5.5 \%)$ did not have documentation of the amount of ILE given. One patient (2.8\%) did not receive a bolus, but was started on an infusion.

\section{Primary results}

The primary outcome of our study was to evaluate the change in MAP over time following the administration of ILE. Data were not collected at the same time points for each patient; for some patients only one measurement was available as death occurred shortly after ILE administration, whereas for other patients, values up to 72 hours after ILE administration were collected. The MAP varied considerably over time and among patients (Figure 1). When all available MAP measurements in the first hour were used in the mixed linear regression model, the MAP at one hour increased by an estimated $13.79 \mathrm{~mm} \mathrm{Hg}$ (95\% CI 1.43-26.15). When patients had the last available single MAP value carried forward to one hour, the mean change in MAP was $17.22 \mathrm{~mm} \mathrm{Hg}$ $(\mathrm{n}=23$; median $=13.33 ; p=0.044$ for one-sided $t$-test assessing MAP $\geq 10 \mathrm{~mm} \mathrm{Hg}$ ). Thirteen $(56.6 \%)$ of these 23 patients had at least a $10 \mathrm{~mm} \mathrm{Hg}$ increase in

\begin{tabular}{|c|c|c|}
\hline Characteristic & Survivors $(n=25)$ & Non-survivors $(n=11)$ \\
\hline Median age (years) $\left(25^{\text {th }}\right.$ percentile, $75^{\text {th }}$ percentile) & $50(30,61)$ & $46(41,55)$ \\
\hline Gender, female, no. (\%) & $11(44.0 \%)$ & $5(45.4 \%)$ \\
\hline Cardiac toxin, no. (\%) & $7(28.0 \%)$ & $3(27.3 \%)$ \\
\hline TCA, no. (\%) & $3(12.0 \%)$ & $2(18.1 \%)$ \\
\hline Bupropion, no. (\%) & $3(12.0 \%)$ & $0(0.0 \%)$ \\
\hline Antiepileptics, no. (\%) & $0(0.0 \%)$ & $1(9.0 \%)$ \\
\hline Mixed Ingestion, no. (\%) & $12(48.0 \%)$ & $5(45.4 \%)$ \\
\hline ACLS care provided, no. (\%) & $3(12.0 \%)$ & $8(72.3 \%)$ \\
\hline Cardiac arrest on presentation & $2(8.0 \%)$ & $4(36.3 \%)$ \\
\hline $\mathrm{SBP}<90 \mathrm{~mm} \mathrm{Hg}$ on presentation, no. (\%) & $8(32.0 \%)$ & $8(72.7 \%)$ \\
\hline $\mathrm{DBP}<60 \mathrm{~mm} \mathrm{Hg}$ on presentation, no. (\%) & $8(33.3 \%)$ & $11(100 \%)$ \\
\hline HR $<50$ bpm on presentation, no. (\%) & $3(12.0 \%)$ & $4(44.4 \%)$ \\
\hline Altered LOC/seizure activity & $8(32.0 \%)$ & $2(18.1 \%)$ \\
\hline Gastric lavage, no. (\%) & $0(0.0 \%)$ & $2(18.1 \%)$ \\
\hline Activated charcoal, no. (\%) & $10(40.0 \%)$ & $5(45.4 \%)$ \\
\hline Whole bowel irrigation, no. (\%) & $5(20.0 \%)$ & $2(18.1 \%)$ \\
\hline Intravenous crystalloid, no. (\%) & $25(100 \%)$ & $11(100 \%)$ \\
\hline Atropine, no. (\%) & $6(24.0 \%)$ & $3(27.3 \%)$ \\
\hline Glucagon, no. (\%) & $13(52.0 \%)$ & $4(36.4 \%)$ \\
\hline Calcium, no. (\%) & $13(52.0 \%)$ & $7(63.6 \%)$ \\
\hline HIE, no. (\%) & $9(36.0 \%)$ & $4(36.4 \%)$ \\
\hline Vasopressors, no. (\%) & $12(48.0 \%)$ & $8(72.3 \%)$ \\
\hline Bicarbonate, no. (\%) & $14(56.0 \%)$ & $9(81.8 \%)$ \\
\hline
\end{tabular}




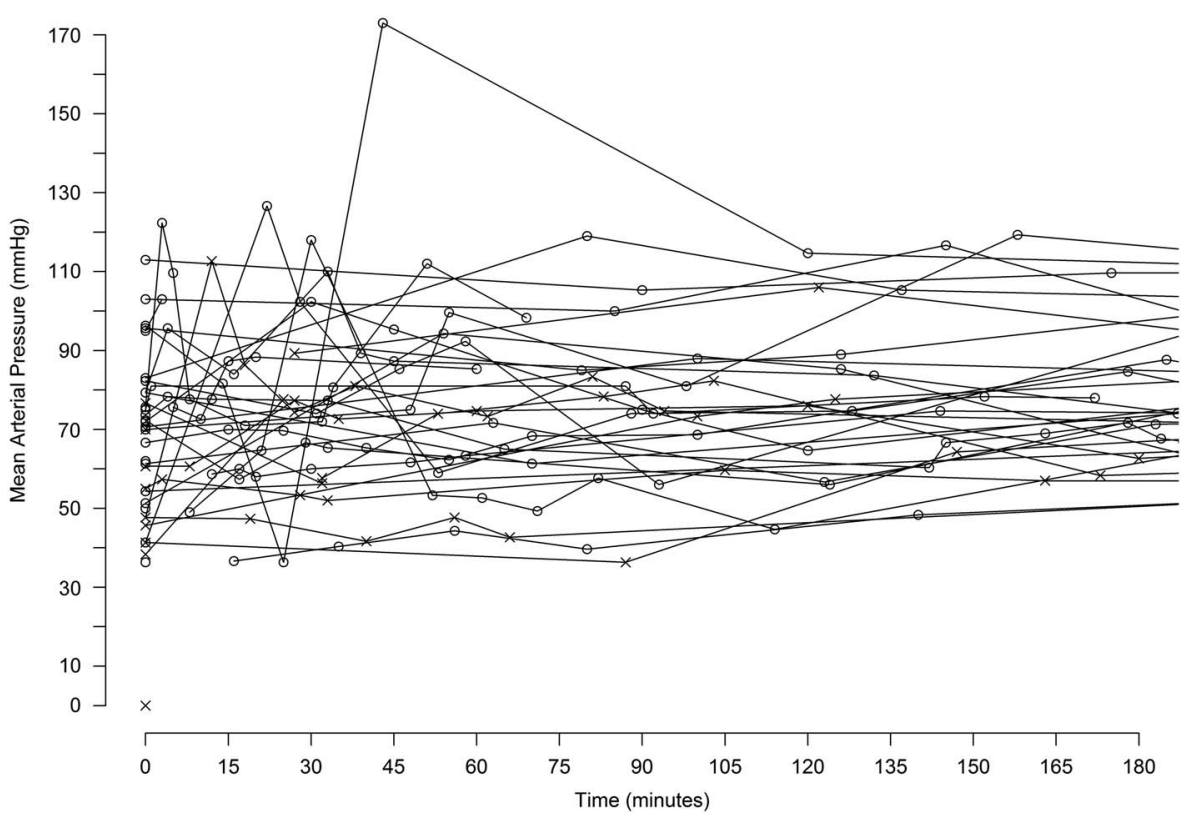

Figure 1. Mean Arterial Pressure Over Time in All Patients

MAP at one hour after ILE administration $(p=0.677)$. This mean change in MAP was highly influenced by one extreme value for one patient (MAP increased by $76.7 \mathrm{~mm} \mathrm{Hg}$ at 43 minutes after ILE administration); without this patient's result, the mean change in MAP was $14.52 \mathrm{~mm} \mathrm{Hg}$ at one hour and was no longer statistically significant $($ median $=11.67 ; p=0.085)$. When survivors were analyzed separately, there was no evidence of a statistically significant increase in MAP in the first hour (mean change $=9.83 \mathrm{~mm} \mathrm{Hg} ; 95 \% \mathrm{CI}$ -4.34-23.99; $\mathrm{n}=25 ; p=0.180$ ) (Figures $2 \mathrm{a}$ and $2 \mathrm{~b}$ ). When baseline MAP values were analyzed for survivors and non-survivors, survivors had a significantly higher mean MAP (73.3 vs. $43.9 \mathrm{~mm} \mathrm{Hg} ; p=0.003$ ). For all analyses with all patients and the survivor and nonsurvivor subgroups, there was no evidence of a statistically significant difference between ingested agent classes or between older and younger patients (age $<65$ and age $\geq 65$ ).

\section{Secondary results}

Overall, 25 (69.0\%) patients survived, with 22 (88.0\%) of these patients regaining their baseline neurologic status at discharge. There was insufficient data to determine the effect of ILE on the time from initiation to discontinuation of vasopressors and inotropes. For survivors, the average ICU length of stay was 6.4 days (median 4, IQR 6), and the average hospital length of stay was
21.4 days (median 10, IQR 15) (Table 2). Drug class did not have a significant effect on patient outcome.

\section{Other findings}

One patient was found to require increased sedation after the administration of ILE. This patient ingested both bupropion and ethanol and became increasingly agitated with seizure activity approximately 48 hours after ingestion. The patient was treated with ILE at this point and found to have worsening agitation shortly after ILE administration.

In addition, interpretation of laboratory values was affected in seven patients (19.4\%). Four of these patients had non-interpretable CBC results due to "grossly lipemic" samples. One other patient had a low-density lipoprotein (LDL) level that could not be interpreted, and one patient had a troponin level that could not be interpreted.

\section{DISCUSSION}

Although our population demonstrated an increase in MAP greater than zero in the first hour after ILE administration, this increase was not statistically significantly greater than the threshold of $10 \mathrm{~mm} \mathrm{Hg}$. In addition, although non-survivors highly influenced our results, when survivors were analyzed separately, the change in MAP remained statistically insignificant. Given 


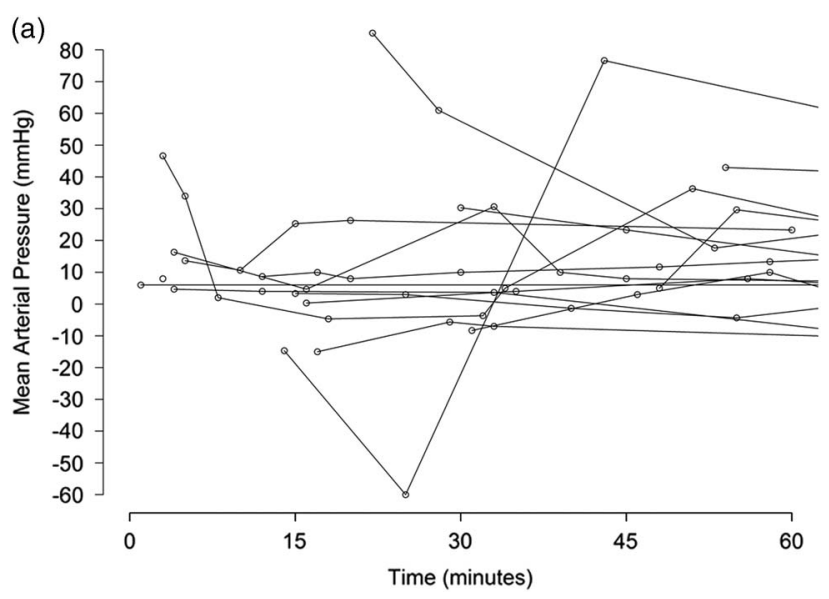

Figure 2a. Change in Mean Arterial Pressure in Survivors

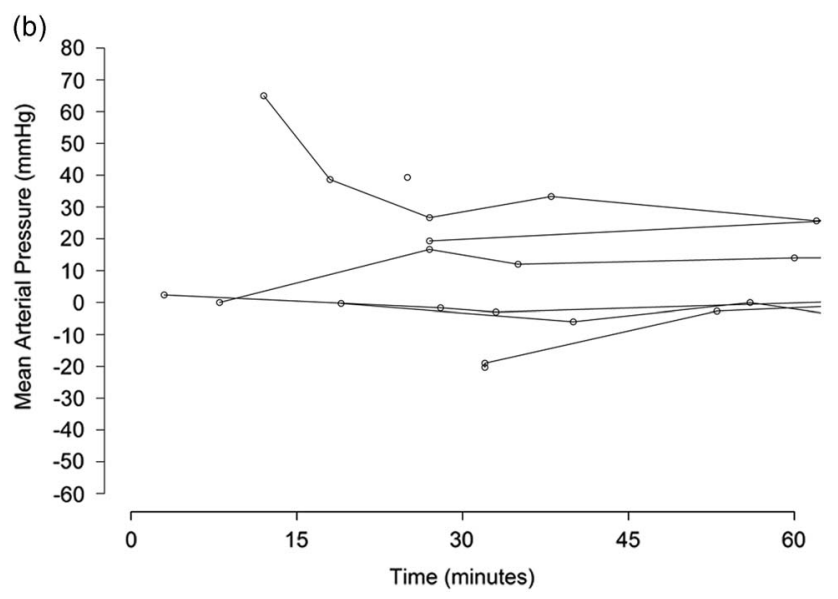

Figure 2b. Change in Mean Arterial Pressure in NonSurvivors

\begin{tabular}{lcc|}
\hline \multicolumn{3}{l}{ Table 2. Summary of outcome measures in study patients } \\
\hline Outcome & Survivors & Non-survivors \\
\hline $\begin{array}{l}\text { Patients with laboratory values } \\
\text { affected by ILE, no }(\%)\end{array}$ & $4(16.0 \%)$ & $2(18.1 \%)$ \\
$\begin{array}{l}\text { Median ICU LOS (days) } \\
(25 \% \text { ile, } 75 \% \text { ile) }\end{array}$ & $4(2,8)$ & $\mathrm{N} / \mathrm{A}$ \\
$\begin{array}{l}\text { Median hospital LOS (days) } \\
(25 \% \text { ile, } 75 \% \text { ile) }\end{array}$ & $10(5,22)$ & $\mathrm{N} / \mathrm{A}$ \\
$\begin{array}{l}\text { Patients returning to baseline } \\
\text { neurologic status, no (\%) }\end{array}$ & $22(88.0 \%)$ & $\mathrm{N} / \mathrm{A}$ \\
\hline
\end{tabular}

ILE: intravenous lipid emulsion; no: number; ICU: intensive care unit; LOS: length of stay; 25\% ile: twenty-fifth percentile; $75 \%$ ile: seventy-fifth percentile

that we examined the change in MAP in the first hour, this non-survivor effect is likely secondary to absolute MAP values in this population being significantly lower on presentation. As a result, the change in MAP in the first hour may have been more pronounced in non-survivors.
These findings are in contrast to several animal and human studies that have demonstrated hemodynamic benefit with ILE administration in various drug classes, including calcium channel blockers, ${ }^{19,23,24}$ amiodarone, ${ }^{48}$ betablockers, ${ }^{15}$ and tricyclic antidepressants. ${ }^{20,26,28}$ The observational nature of our study, compared to the randomized, controlled design of many of the animal and human studies, is the most likely explanation for the difference.

Almost all of the survivors regained their baseline neurologic status prior to hospital discharge. This outcome has not been studied in previously published reports on the use of ILE. Although an association between ILE administration and this finding cannot be extrapolated from our study, this relationship could be explored further in future studies.

One patient with a bupropion and ethanol overdose was noted to be difficult to sedate after ILE administration; this may be secondary to the lipophilic nature of sedative agents being adsorbed into the ILE. Although this may represent a novel adverse event, it could also be explained by worsening ethanol withdrawal, given the timeline of this patient's increased agitation and seizure activity. If this was, in fact, a novel adverse event, there are several sedative and analgesic agents for which this may be relevant, including fentanyl, midazolam, etomidate, propofol, phenobarbital, and ketamine.

Prior studies have identified difficulty with interpreting laboratory investigations after ILE administration, such as liver transaminases, serum lipid profile, and some electrolytes. ${ }^{51,52}$ In our study, the most commonly reported interference from ILE was with the complete blood count. One animal study demonstrated that ILE interferes with lipid profile lab results, ${ }^{53}$ which was seen with one of our patients. To our knowledge, there has not been any previous report of ILE interfering with troponin value interpretation.

Our results are similar to those reported by Geib et al., ${ }^{49}$ who studied the use of ILE in cardiac arrest patients. Like this study, ours was unable to demonstrate a clinically significant improvement in MAP following ILE administration. Furthermore, both Geib et al. $^{49}$ and Levine et al. ${ }^{50}$ reported multiple adverse effects in a large majority of the patients studied, including ARDS and pancreatitis. In our study, we did not systematically collect adverse event data; this is the most likely reason that our data differs from the above studies.

This study represents the largest human case series on the use of ILE in non-local anesthetic overdoses. Prior studies supporting the effectiveness of ILE in humans 
have been limited to case reports, case series, and review articles. Human data, in the form of single case reports, are subject to publication bias with negative case reports much less likely to be published. Unlike previously published human case reports, we enrolled consecutive patients that received ILE therapy and our results included patients that survived or died after the administration of ILE. The majority of published case reports on the utility of ILE in this setting have positive outcomes, ${ }^{22-32}$ although negative case reports on the effect of ILE in overdose patients have also been published. ${ }^{54,55}$

\section{Limitations}

Our study has several limitations. Given the observational study design, claims on efficacy (or lack of efficacy) of ILE as an antidote cannot be made. Only patients where the treating health care provider contacted our poison center were enrolled. It is possible that eligible patients for whom the poison center was not consulted were missed.

Another limitation is the variability in the dosing protocols of ILE. Our poison center's dosing protocol was made available to regional EDs and ICUs, and is based on the widely accepted ILE dosing protocols. ${ }^{41}$ This protocol was also available on the promotional posters distributed prior to patient enrolment. Since many patients had already been started on ILE by the time our poison center was contacted, some variability in the dose administered was expected. During our study period, there was also a regional change in ILE formulation from Intralipid ${ }^{\circledR}$ to Clinoleic ${ }^{\circledR}$. There was no evidence of statistically significant effect on death with this change $(p=0.46)$. Furthermore, although our population closely reflects the heterogeneous population encountered in the emergency department, this heterogeneity may limit the validity of our results.

In addition, because some data were collected retrospectively, we were dependent on the information available within the medical record. As a result, some data were missing. The utilization of the CSPIs at our poison center allowed for regular telephone follow-up and collection of patient data where available. However, the initial critical nature of most patients, the early death of some patients, and the prolonged hospital stay of several patients made these data difficult to collect regularly, particularly the documentation outlining the start and cessation of vasopressor and inotropic agents.
Finally, our adverse event data was also limited. We did not systematically collect lipase levels or examine chest $\mathrm{x}$-ray results on each patient, but rather examined charts retrospectively for any evidence of possible adverse events. This may have resulted in adverse events being missed.

\section{CONCLUSION}

In this cohort of hemodynamically unstable poisoned patients, no clinically significant improvement in MAP occurred after ILE administration. Adverse events included the need for increased sedation after ILE administration in one patient and interference with lab analyses in six patients. Future research involving ILE should include prospective, randomized controlled trials to more definitively study the efficacy of ILE and to help ascertain the most appropriate dosing protocol. Until then, ILE remains a potential treatment option in hemodynamically unstable overdose patients when conventional therapy has failed.

Acknowledgements: We would like to thank Joanne Masur and Miriam Weber for their contributions in study design and data collection.

We are thankful to both University of Calgary Emergency Medicine Research Advisory Committee and the Poison and Drug Information Service for providing grant funding for the study. Dr. Rosychuk is salary supported by Alberta Innovates-Health Solutions (AI-HS; Edmonton, Canada) as a Health Scholar.

Competing Interests: All authors have declared that they hold no conflicts of interest of any kind in relation to this submission. The views expressed in this article are solely those of the authors and are not an official position of the University of Alberta or University of Calgary.

\section{REFERENCES}

1. Weinberg GL, VadeBoncouer T, Ramaraju GA, et al. Pretreatment or resuscitation with a lipid infusion shifts the dose-response to bupivacaine-induced asystole in rats. Anesthesiology 1998;88(4):1071-5.

2. Zausig YA, Zink W, Keil M, et al. Lipid emulsion improves recovery from bupivacaine-induced cardiac arrest, but not from ropivacaine- or mepivacaine-induced cardiac arrest. Anesth Analg 2009;109(4):1323-6.

3. Aprea F, Vettorato E, Corletto F. Severe cardiovascular depression in a cat following a mandibular nerve block with bupivacaine. Vet Anaesth Analg 2011;38(6):614-8.

4. Bushey BA, Auld VH, Volk JE, et al. Combined lipid emulsion and ACLS resuscitation following bupivacaine-and hypoxia-induced cardiovascular collapse in unanesthetized swine. AANA7 2011;79(2):129-38. 
5. Weinberg G, Ripper R, Feinstein DL, et al. Lipid emulsion infusion rescues dogs from bupivacaine-induced cardiac toxicity. Reg Anesth Pain Med 2003;28(3):198-202.

6. Admani B, Essajee F. Successful resuscitation of a three month old child with intralipid infusion, presumed to have bupivacaine induced seizures and cardiovascular complications: case report. East Afr Med $\mathcal{7}$ 2010;87(8):354-6.

7. Bourne E, Wright C, Royse C. A review of local anesthetic cardiotoxicity and treatment with lipid emulsion. Local Reg Anesth 2010;3:11-9.

8. Hoegberg LCG, Bania TC, Lavergne V, et al. Systematic review of the effect of intravenous lipid emulsion therapy for local anesthetic toxicity. Clin Toxicol 2016, doi: $10.3109 / 15563650.2015 .1121270$.

9. Neal JM, Bernards CM, Butterworth JF, et al. ASRA practice advisory on local anesthetic systemic toxicity. Reg Anesth Pain Med 2010;35(2):152-61.

10. Kuo I, Akpa BS. Validity of the lipid sink as a mechanism for the reversal of local anesthetic systemic toxicity: a physiologically based pharmacokinetic model study. Anesthesiology 2013;118(6): 1350-61.

11. Weinberg GL. Lipid resuscitation: more than a sink. Crit Care Med 2012;40(8):2521-3.

12. French D, Smollin C, Ruan W, et al. Partition constant and volume of distribution as predictors of clinical efficacy of lipid rescue for toxicological emergencies. Clin Toxicol 2011;49:801-9.

13. Weinberg GL. Lipid emulsion infusion: resuscitation for local anesthetic and other drug overdose. Anesthesiology 2012;117(1):180-7.

14. Lee SH, Sung H, Ok S, et al. Lipid emulsions enhance the norepinephrine-mediated reversal of local anesthetic-induced vasodilation at toxic doses. Yonsei Med 7 2013;54(6):1524-32.

15. Cave G, Harvey M. Lipid emulsion may augment early blood pressure recovery in a rabbit model of atenolol toxicity. 7 Med Toxicol 2009;5(1):50-1.

16. Browne A, Harvey M, Cave G. Intravenous lipid emulsion does not augment blood pressure recovery in a rabbit model of metoprolol toxicity. 7 Med Toxicol 2010;6(4):373-8.

17. Harvey M, Cave G, Lahner D, et al. Insulin versus Lipid Emulsion in a Rabbit Model of Severe Propranolol Toxicity: A Pilot Study. Crit Care Research and practice 2011; (2011):361737.

18. Hayes CL, Knight M. Calcium channel blocker toxicity in dogs and cats. The Veterinary clinics of North America Small animal practice 2012;42(2):263-77.

19. Bania TC, Chu J, Perez E, et al. Hemodynamic effects of intravenous fat emulsion in an animal model of severe verapamil toxicity resuscitated with atropine, calcium, and saline. Academic emergency medicine 2007;14(2):105-11.

20. Heinonen JA, Litonius E, Backman JT, et al. Intravenous lipid emulsion entraps amitriptyline into plasma and can lower its brain concentration - an experimental intoxication study in pigs. Basic \& Clinical Pharmacology \& Toxicology 2013;113(3):193-200.

21. Harvey M, Cave G. Intralipid outperforms sodium bicarbonate in a rabbit model of clomipramine toxicity. Ann Emerg Med 2007;49(2):178-85.

22. Patel T, Tietze D, Mehta AN. Amlodipine overdose. Proceedings (Baylor University Medical Center) 2013;26(4):410-1.
23. Bologa C, Lionte C, Coman A, et al. Lipid emulsion therapy in cardiodepressive syndrome after diltiazem overdose - case report. Am 7 Emerg Med 2013;31(7):1154.e3-4.

24. Liang CW, Diamond SJ, Hagg DS. Lipid rescue of massive verapamil overdose: a case report. Fournal of medical case reports 2011;5(1):399.

25. Akinci E, Koylu R. Verapamil Poisoning, the Importance of Intravenous Lipid Therapy: Case Report. Fournal of Academic Emergency Medicine Case Reports 2013;4:130-2.

26. Engels PT, Davidow JS. Intravenous fat emulsion to reverse haemodynamic instability from intentional amitriptyline overdose. Resuscitation 2010;81(8):1037-9.

27. Boegevig S, Rothe A, Tfelt-Hansen J, et al. Successful reversal of life threatening cardiac effect following dosulepin overdose using intravenous lipid emulsion. Clinical toxicology 2011;49(4):337-9.

28. Hendron D, Menagh G, Sandilands EA, et al. Tricyclic antidepressant overdose in a toddler treated with intravenous lipid emulsion. Pediatrics 2011;128(6):e1628-32.

29. Livshits Z, Feng Q, Chowdhury F, et al. Life-threatening bupropion ingestion: is there a role for intravenous fat emulsion? Basic \& Clinical Pharmacology \& Toxicology 2011;109(5):418-22.

30. Jakkala-Saibaba R, Morgan PG, Morton GL. Treatment of cocaine overdose with lipid emulsion. Anaesthesia 2011;66 (12):1168-70.

31. Finn SDH, Uncles DR, Willers J, et al. Early treatment of a quetiapine and sertraline overdose with Intralipid. Anaesthesia 2009;64(2):191-4.

32. Cave G, Harvey M, Willers J, et al. LIPAEMIC report: results of clinical use of intravenous lipid emulsion in drug toxicity reported to an online lipid registry. Fournal of Medical Toxicology 2014;10(2):133-42.

33. Levine M, Hoffman RS, Lavergne V, et al. Systematic review on the effect of intravenous lipid emulsion therapy for non-local anesthetics toxicity. Clinical Toxicology 2016; doi: $10.3109 / 15563650.2015 .1126286$.

34. Kasi VS, Estrada CA, Wiese W. Association of pancreatitis with administration of contrast medium and intravenous lipid emulsion in a patient with the acquired immunodeficiency syndrome. South Med 7 2003;96(1):66-9.

35. Raasch RH, Hak LJ, Benaim V, et al. Effect of intravenous fat emulsion on experimental acute pancreatitis. Fournal of Parenteral Nutrition 1983;7(3):254-6.

36. Weinberg G. Hyperamylasemia following lipid resuscitation: pancreas or parotid? Anesth Analg 2012;115(3):739.

37. Bass J, Friedl $W$, Jeranek $W$. Intralipid causing adult respiratory distress syndrome. 7 Natl Med Assoc 1984;76(4):401-3.

38. Ali J, Wood L. The acute effects of intralipid on lung function. 7 Surg Res 1985;38(6):599-605.

39. Koch T, Duncker HP, Klein A, et al. Modulation of pulmonary vascular resistance and edema formation by short-term infusion of a $10 \%$ fish oil emulsion. Infusionsther Transfusionsmed 1993;20(6):291-300.

40. Ishitsuka $\mathrm{Y}$, Moriuchi $\mathrm{H}$, Yang $\mathrm{C}$, et al. Effects of bolus injection of soybean-based fat emulsion and fatty acids on pulmonary gas exchange function. Biol Pharm Bull 2009;32(3):500-3.

41. Calmarza P, Cordero J. Lipemia interferences in routine clinical biochemical tests. Biochemia medica 2011;21(2):160-6. 
42. Grunbaum AM, Gilfix BM, Gosselin S, et al. Analytical interferences resulting from intravenous lipid emulsion. Clin Toxicol 2012;50(9):812-7.

43. Johnson-Arbor K, Salinger L, Luczycki S. Prolonged Laboratory Interference After Administration of Intravenous Lipid Emulsion Therapy. 7 Med Toxicol 2015;11(2):223-6.

44. American College of Medical Toxicology. ACMT position statement: interim guidance for the use of lipid resuscitation therapy. 7 Med Toxicol 2011;7:81-2.

45. LipidRescue ${ }^{\mathrm{TM}}$ Resucitation for drug toxicity. Treatment Regimens. Available at: http://www.lipidrescue.org (accessed December 11, 2009).

46. Florian JP, Pawelczyk JA. Non-esterified fatty acids increase arterial pressure via central sympathetic activation in humans. Clin Sci 2010;118:61-9.

47. Pinheiro J, Bates D, DebRoy S, et al. 2015. nlme: Linear and Nonlinear Mixed Effects Models. $R$ package version, 3:1-122; Available at: http://CRAN.R-project.org/package=nlme.

48. Niiya $T$, Litonius $E$, Petaja $L$, et al. Intravenous lipid emulsion sequesters amiodarone in plasma and eliminates its hypotensive action in pigs. Ann Emerg Med 2010;56(4): 402-8.

49. Geib A, Liebelt E, Manini AF. Clinical experience with intravenous lipid emulsion for drug-induced cardiovascular collapse. 7 Med Toxicol 2012;8(1):10-4.

50. Levine M, Skolnik AB, Ruha A, et al. Complications following antidotal use of intravenous lipid emulsion therapy. $7 \mathrm{Med}$ Toxicol 2014;10:10-4.

51. Punja M, Neill SG, Wong S. Caution with interpreting laboratory results after lipid rescue therapy. Am 7 Emerg Med 2013;31(10):1536.e1-2.

52. Kroll MH. Evaluating interference caused by lipemia. Clin Chem 2004;50(11):1968-9.
53. Reimold EW. Studies of the toxicity of an intravenous fat emulsion. II. Blood chemical changes after administration of a soybean oil (FE-S15) in beagles. 7 Parenter Enteral Nutr 1979;3(5):335-40.

54. Miller S, Greenberg M. Failure of lipid emulsion therapy to treat a metformin overdose. Clin Toxicol 2011;49 (6):538-9.

55. Litonius E, Niiya T, Neuvonen PJ, et al. No antidotal effect of intravenous lipid emulsion in experimental amitriptyline intoxication despite significant entrapment of amitriptyline. Basic Clin Pharm Toxicol 2012;110(4):378-83.

\section{APPENDIX 1}

\section{Threshold systolic blood pressure}

$\begin{array}{ll}<1 \text { month } & <60 \\ 1 \text { month- } 1 \text { year } & <70 \\ 1-10 \text { years } & <70 \\ >10 \text { years } & <90\end{array}$

Threshold heart rate

\begin{tabular}{lr}
\hline Newborn-3 months & $<85$ \\
3 months-2 years & $<100$ \\
2 years- 10 years & $<60$ \\
$>10$ years & $<60$ \\
\hline
\end{tabular}

